\title{
Identical cytokeratin expression pattern CK7 + /CK20 - in esophageal and cardiac cancer: etiopathological and clinical implications
}

\author{
A Driessen ${ }^{1}, \mathrm{P}$ Nafteux ${ }^{2}, \mathrm{~T}_{\text {Lerut }}^{2}$, D Van Raemdonck ${ }^{2}, \mathrm{P}$ De Leyn ${ }^{2}, \mathrm{~L}$ Filez ${ }^{3}, \mathrm{~F}$ Penninckx ${ }^{3}$, \\ $\mathrm{K}_{\text {Geboes }}{ }^{4}$ and N Ectors ${ }^{4}$ \\ ${ }^{1}$ Department of Pathology, University Hospital Maastricht, The Netherlands; ${ }^{2}$ Department of Thoracic \\ Surgery; ${ }^{3}$ Department of Abdominal Surgery and ${ }^{4}$ Department of Pathology, University Hospitals Leuven, \\ Belgium
}

\begin{abstract}
Surgical treatment and prognosis is different in esophageal, cardiac and distal gastric adenocarcinomas. Determination of the origin, in particular of adenocarcinomas situated at the gastroesophageal junction, may be difficult. It has been suggested that esophageal adenocarcinomas are characterized by a specific cytokeratin pattern, namely the CK7 + /CK20 - pattern. According to the same authors, this cytokeratin pattern is absent in gastric adenocarcinomas. The aim of our study is to evaluate if this cytokeratin pattern CK7 +/CK20 - is absent in cardiac and distal gastric adenocarcinomas. Therefore, we evaluated the combined immunohistochemical expression of CK7 and CK20 on paraffin-embedded material of 214 resection specimens for adenocarcinoma, comprising 66 esophageal, 73 cardiac and 75 distal gastric adenocarcinomas (UICC-classification). The adenocarcinomas were subtyped into intestinal- and diffuse-type according to the Lauren classification. The immunohistochemical staining was considered as positive if $\mathbf{5 0 \%}$ or more of the tumor cells were stained. Statistical analysis has been performed applying the $\chi^{2}$-test. The tumors situated at the gastroesophageal junction, esophageal as well as cardiac adenocarcinomas, showed predominantly a CK7 + /CK20-expression pattern (67 vs $68 \%$ ), whereas this cytokeratin pattern is rather uncommon in distal gastric adenocarcinomas $\left(31 \%, P<4 \times 10^{-5}\right)$. Independent of their localization, intestinal- as well as diffuse-type adenocarcinomas have a similar cytokeratin pattern. Our data show that the combined expression of CK7 and CK20 is different for the adenocarcinomas situated on both sides of the gastroesophageal junction compared to the distal gastric adenocarcinomas. However, in contrast to data in the literature, the combined expression of CK7 and CK20 has a low specificity in the distinction between esophageal and cardiac adenocarcinomas. This may suggest a similar origin (cell lineage) and thus may have an impact on therapeutic strategies.
\end{abstract}

Modern Pathology (2004) 17, 49-55, advance online publication, 19 November 2003; doi:10.1038/modpathol.3800011

Keywords: cytokeratins; gastric adenocarcinoma; esophageal adenocarcinoma; cardiac adenocarcinoma

Epidemiological studies have shown that the topographical distribution of gastric cancer is changing. Tumors become more prevalent in the cardia, whereas the incidence of gastric cancer confined to the distal part of the stomach decreases. ${ }^{1,2}$ During the last few decades the annual age-adjusted incidence of cardiac adenocarcinomas has increased by $4-5 \% .^{3}$ This rise in incidence occurs in parallel

Correspondence: A Driessen, Department of Pathology, University Hospital Maastricht, P Debyelaan 25 Postbus 5800, AZ 6202 Maastricht, The Netherlands.

E-mail: adri@lpat.azm.nl

Received 28 July 2003; revised 04 September 2003; accepted 05

October 2003; published online 19 November 2003 with the increase in incidence of esophageal adenocarcinomas. In white male subjects, the ageadjusted incidence rates of this cancer increased from 0.7 during 1974-1976 to 3.2 during 1992$1994 .^{2}$ The epidemiological study of El-Serag et $a l^{4}$ suggests that these tumors are two distinct entities: the increase in incidence of esophageal and cardiac adenocarcinomas is both related to age, but only in esophageal adenocarcinomas a cohort effect is observed. Although the prognosis of esophageal adenocarcinomas is poor, cardiac adenocarcinomas have an even worse prognosis, due to an advanced stage of disease and the presence of lymph node metastasis at the time of diagnosis. ${ }^{5-8}$ The localization of the tumor, and also the different 
dissemination pattern to the lymph nodes will determine the choice of treatment. There is still some debate about the type and the extent of surgical treatment. To improve the survival of patients, the need for a wide peritumoral dissection, including the removal of peritumoral tissue in the mediastinum, is adviced. Extended lymphadenectomy may improve the overall survival., ${ }^{9,10}$

Moreover, different precursors may be involved in the histogenesis of esophageal adenocarcinomas and cardiac adenocarcinomas. In the first type, the relationship with Barrett's esophagus is wellknown. The development of a Barrett's esophagus is the result of a longstanding reflux of acid and bile. ${ }^{11,12}$ The etiopathogenesis of cardiac adenocarcinomas is less clear. Carditis and intestinal metaplasia are proposed as precursor lesions for this cancer. Several studies identified a relationship between carditis and gastroesophageal reflux. ${ }^{13,14}$ According to other studies, however, carditis was an extension of a Helicobacter pylori-related pangastritis. ${ }^{15,16}$

Based on these epidemiological and etiopathological data, distal esophageal and cardiac adenocarcinomas would seem to be two distinct entities. However, both adenocarcinomas mostly present in an advanced stage of disease, as a result of which, the origin of these tumors is difficult to determine. According to Ormsby et $a l,{ }^{17}$ Barrett's related esophageal adenocarcinomas are characterized by a specific cytokeratin pattern, namely the CK7 + / CK20- expression pattern. This pattern would seem to be confined to this type of tumor and its precursor, when compared to gastric cancer. However, the number of gastric adenocarcinomas $(n=14)$ investigated in their study was limited. Furthermore, these gastric tumors originated from the subcardia and by definition not cardia, as only these tumors were included of which the most proximal part of the tumor was situated at a minimum distance of $35 \mathrm{~mm}$ of the gastroesophageal junction. Moreover, they only studied intestinaltype of adenocarcinomas.

Therefore, we performed a similar study on a larger number of cases, originating from the esophagus, cardia and distal stomach, divided into intestinal- and diffuse-type using the same methodology. The aim of our study was to evaluate whether this CK7 + /CK20 - pattern was confined to esophageal adenocarcinomas.

\section{Materials and methods}

We have reviewed 214 adenocarcinomas of the esophagus, the cardia or the distal stomach (Table 1). Adenocarcinomas situated at the gastroesophageal junction were classified into esophageal or cardiac adenocarcinomas according to recommendations of the International Union Against Cancer (UICC). The major criteria for the distinction between gastric cardia and distal esophagus were the localization of the bulk of the tumor and the presence of an associated Barrett's esophagus. If more than $50 \%$ of the mass of the tumor was situated in the cardia, the tumor was classified as being of cardiac origin, whereas if the mass of the tumor was predominantly found in the esophagus, the UICC advises to classify it as an esophageal tumor. According to these advices, a tumor situated on the gastroesophageal junction was likely to be of esophageal origin when the neoplastic lesion was associated with a Barrett's esophagus of the specialized or intestinal type. ${ }^{18}$ The presence of a Barrett's esophagus was evaluated on biopsies taken above the gastro-esophageal junction, either during endoscopic examinations prior to surgery, and/or from the resection specimen. Barrett's esophagus was defined by the presence of intestinal type of columnar epithelium independent of the length of the lesion. ${ }^{19}$ Distal gastric tumors were situated in the corpus and/or the antrum of the stomach.

Routine histology was performed on samples of the resection specimen, fixed in formaldehyde and embedded in paraffin. In accordance with the Lauren classification, the tumors were classified into diffuse- or intestinal-type of adenocarcinomas, based on their histopathological features. Tumors demonstrating mixed histological features were classified according their predominant features. ${ }^{20}$

In order to evaluate the presence of goblet cells in the epithelium of a Barrett's esophagus, an alcian blue/PAS staining ( $\mathrm{pH}$ 2.5) was performed. This stain allows distinction between gastric (red) and intestinal type (blue) of mucins. ${ }^{21}$

Immunohistochemistry was performed on one tissue block, representative of the tumor mass in the resection specimen. For immunohistochemical analysis, we used a CK7-antibody (Dako, Denmark, monoclonal antibody, clone OV-TL 12/30, dilution $1 / 50$ ) and a CK20-antibody (Dako, Denmark, monoclonal antibody, clone Ks20.8, dilution 1/50), on semiserial sections of the paraffin-embedded, formol-fixed material. The staining carried out using an avidin-biotin method with antigen retrieval. The staining was performed on an automated Ventana IHC instrument, using the Ventana basic DAB detection kit. Our immunohistochemical analysis was validated through positive and negative controls (by omitting the primary antibody). In accordance with the criteria of Ormsby et a $1^{17}$

Table 1 Clinical data of the patients

\begin{tabular}{lccc}
\hline & $\begin{array}{c}\text { Esophageal } \\
\text { cancer }\end{array}$ & $\begin{array}{c}\text { Cardiac } \\
\text { cancer }\end{array}$ & $\begin{array}{c}\text { Distal gastric } \\
\text { cancer }\end{array}$ \\
\hline Number of cases & 66 & 73 & 75 \\
Mean age (years) & 65 & 64 & 70 \\
Range (years) & $32-86$ & $37-82$ & $41-90$ \\
M/F ratio & 7.25 & 4.21 & 1.88 \\
\hline
\end{tabular}


a tumor was considered to be positive for CK7 or CK20 if $50 \%$ or more of the tumor cells showed a predominantly intense cytoplasmic positivity for this cytokeratin.

For statistical analysis, we used the $\chi^{2}$-test, in which $P<0.05$ was considered to be statistically significant. The sensitivity, the specificity and the positive predictive value were determined according to the Bayes theorem.

\section{Results}

\section{Clinical Data of the Patients}

The series of 214 patients, was composed of 66 patients, having a distal esophageal adenocarcinoma, 73 patients with a cardiac carcinoma and 75 patients with a distal gastric cancer. The mean age of the overall population was 66 years (range 3290 years). Table 1 presents the clinical characteristics of all patients included in this study. Whereas the group of esophageal and cardiac carcinomas have a comparable age distribution, the group of patients, having a distal gastric cancer, consists of elderly people. In this group, there is no significant gender predominance, whereas the groups of esophageal adenocarcinomas $(P<0.003)$ and cardiac adenocarcinomas $(P<0.05)$ are both mainly composed of males.

\section{Immunohistochemical Analysis in Function of the Localization of the Adenocarcinomas (Figure 1)}

Immunohistochemical analysis revealed a heterogeneous combined expression pattern of CK7 and CK20. The overall prevalence of CK7 - positivity was $66 \%(n=142 / 214)$ with the following distribution pattern: esophageal adenocarcinomas $82 \% \quad(n=54 /$ 66), cardiac adenocarcinomas 77\% $(n=57 / 73)$ and distal gastric adenocarcinomas $39 \% \quad(n=31 / 75)$. There was a significant lower prevalence in distal gastric carcinomas than in esophageal $\left(P=2 \times 10^{-6}\right)$ and cardiac carcinomas $\left(P=1 \times 10^{-5}\right)$. CK20 immunoreactivity was present in $18 \%$ of the whole population $(n=38 / 214)$. The distribution of

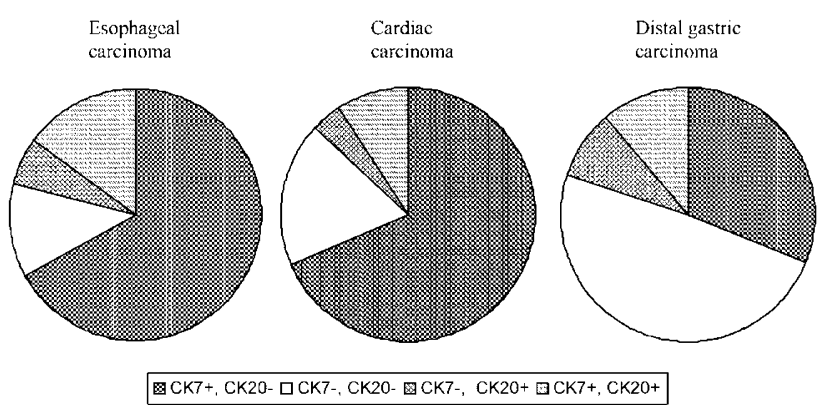

Figure 1 Distribution of the expression pattern of CK7 and CK20 in function of the localization of the carcinomas.
CK20-positivity in function of the location of the tumor did not vary significantly: esophageal adenocarcinomas $21 \% \quad(n=14 / 66)$, cardiac adenocarcinomas $13 \% \quad(n=10 / 73)$ and distal gastric adenocarcinomas $20 \%(n=15 / 75)$. Esophageal and cardiac adenocarcinomas were mostly characterized by a presence of CK7, combined with an absence of CK20 (Figures 2 and 3). The prevalence of this CK7 + /CK20 - pattern is comparable in esophageal $(67 \%, \quad n=44 / 66)$ and cardiac adenocarcinomas $(68 \%, n=50 / 73)$ and is significantly higher than in distal gastric adenocarcinomas (31\%, $n=23 / 75)$ $\left(P=9 \times 10^{-6}\right.$ vs $\left.P=4 \times 10^{-5}\right)$. The sensitivity, the specificity and the positive predictive value of this cytokeratin pattern for esophageal adenocarcinomas was 67,51 and $38 \%$, respectively, which was comparable to those for cardiac adenocarcinomas, namely 68, 52 and $43 \%$, respectively. In contrast, nearly half of the distal gastric adenocarcinomas did not express CK7 or CK20 (49\%, $n=37 / 75)$. This CK7-/CK20- pattern was only observed in $12 \%$ of the esophageal adenocarcinomas $(n=8 / 66)$ $\left(P=5 \times 10^{-6}\right)$ and in $18 \%$ of the cardiac adenocarcinomas $(n=13 / 73)\left(P=1 \times 10^{-4}\right)$. Although the sensitivity of this cytokeratin pattern for distal gastric carcinomas is low, namely $49 \%$, it has a high specificity, namely $81 \%$ and a moderately high positive predictive value, namely $64 \%$.

The frequency of the other cytokeratin patterns, namely $\mathrm{CK} 7+/ \mathrm{CK} 20+$ or $\mathrm{CK} 7-/ \mathrm{CK} 20+$, was independent of the localization of the adenocarcinomas.

\section{Immunohistochemical Analysis in Function of the Type of Adenocarcinomas, Subtyped According to the Lauren Classification (Figure 4)}

Analysis of the morphological features of the tumors showed that our population of adenocarcinomas consisted predominantly of intestinal-type of tumors $(68 \%, n=145 / 214)$, namely in the esophagus $(n=79 \%, 52 / 66)$, the cardia $(73 \%, n=53 / 73)$ and less so in the distal stomach $(53 \%, n=40 / 75)$. The overall prevalence of diffuse-type adenocarcinomas was $32 \%$ with the following distribution pattern: esophagus $21 \%$, cardia $27 \%$ and distal stomach $47 \%$.

A diffuse cytoplasmatic positivity for CK7 was overall diagnosed in $66 \%(n=142 / 214)$ with a nearly significant higher prevalence in intestinaltype adenocarcinomas $70 \% \quad(n=101 / 145)$ than in diffuse-type adenocarcinomas $55 \% \quad(n=38 / 69)$ $(P=0.053)$. CK20-positivity was present in a limited number of intestinal-(19\%, $n=27 / 145)$ and diffuse-type adenocarcinomas (18\%, $n=12 / 69)$. There was no difference in coexpression of CK7 and CK20 in intestinal- and diffuse-type adenocarcinomas. The CK7 + /CK20- pattern was observed in $57 \%$ of the intestinal-type adenocarcinomas $(n=83 / 145)$ and $55 \%$ of the diffuse-type adenocarcinomas $(n=32 / 69)$, whereas the CK7-,CK20- 

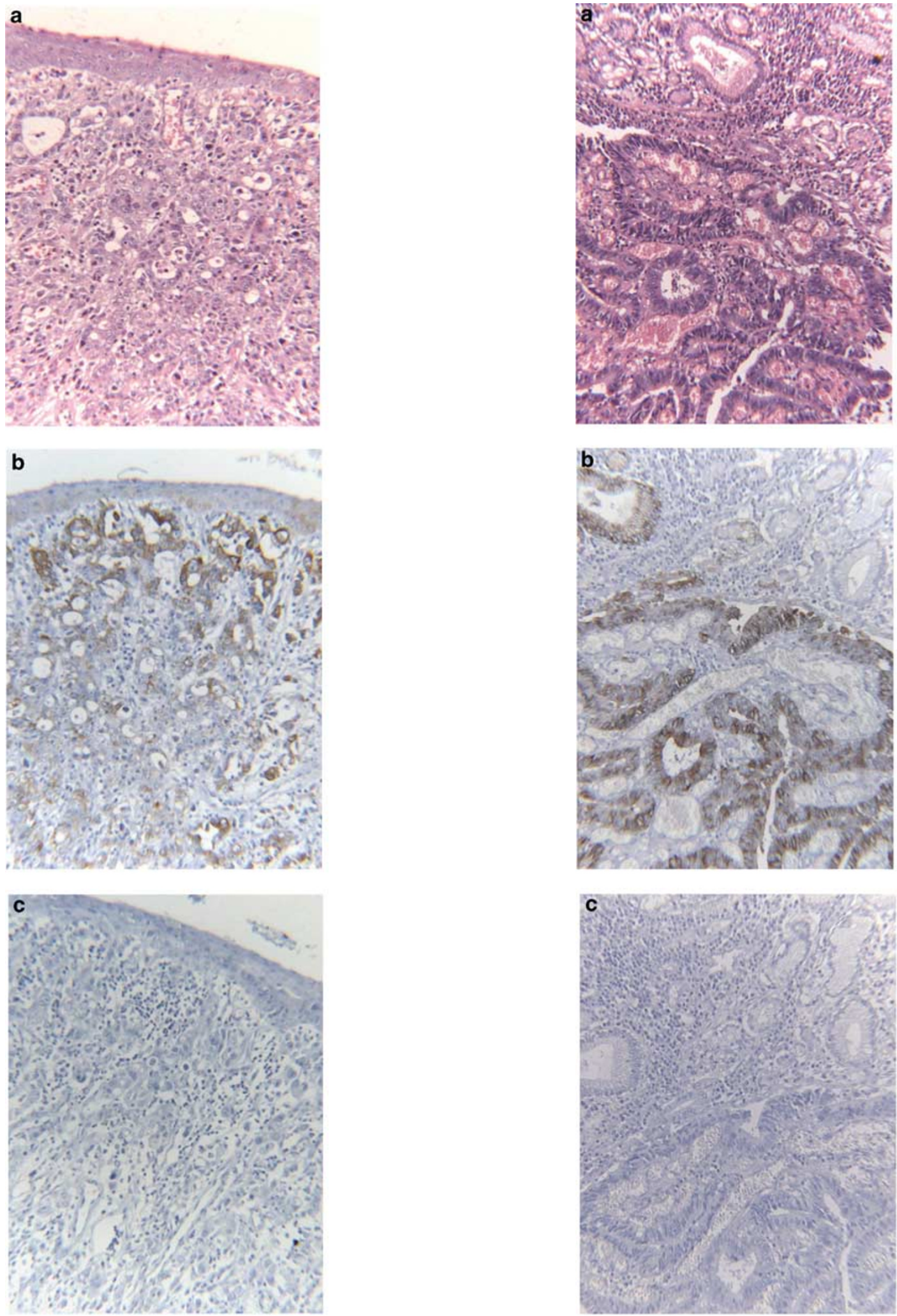

Figure 2 Majority of esophageal adenocarcinomas (H/E, a, x200) are characterized by a combined expression of CK7 (b, x200) and an absence of CK20 (c, x200).

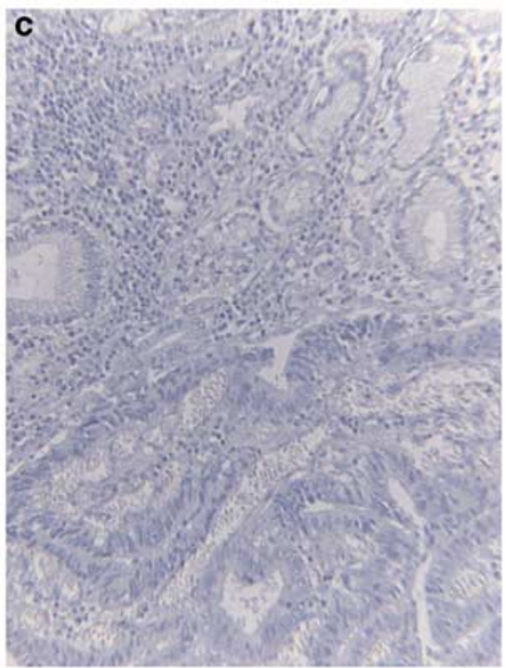

Figure 3 Cytokeratin pattern of cardiac adenocarcinomas (H/E, a, $\mathrm{x} 200$ ) is similar to that in esophageal adenocarcinomas, in which they show a CK7 + (b, x200) and CK20- pattern (c, x200). 


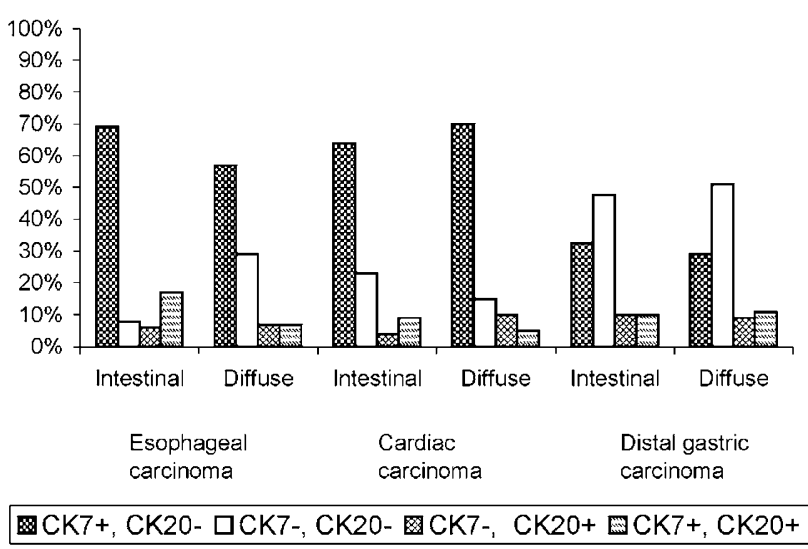

Figure 4 Expression of cytokeratins 7 and 20 in function of the Lauren classification and the localization of the tumor.

pattern was present in $36 \%$ of the diffuse-type adenocarcinomas $(n=25 / 69)$ and in $24 \%$ of the intestinal-type adenocarcinomas $(n=35 / 145)$. The cytokeratin pattern in esophageal and cardiac adenocarcinomas, as well as the distal gastric adenocarcinomas did not vary in function of their histological type (Figure 2).

\section{Discussion}

Immunohistochemistry is currently routinely applied in the diagnosis of human cancer. Antibodies directed against cytokeratins, which are intermediate filament proteins, can establish the epithelial cell lineage of neoplastic cells. Cytokeratins are composed of tetrameric heteropolymers of two different keratins, belonging to the family of acidic (type I) and basic (type II) keratins. ${ }^{22}$ Certain cytokeratins have a restricted tissue distribution. Therefore, they can be used to identify certain subtypes of carcinomas. CK7, a type II cytokeratin with a molecular weight of $54 \mathrm{kDa}$, is strongly expressed in normal tissues such as the transitional cell epithelium of the bladder and the ducts of the pancreatico-biliary system. In function of the applied clone (OV-TL 12/30) the surface epithelium of the stomach can also express CK7. ${ }^{23}$ CK20, a type I cytokeratin with a molecular weight of $46 \mathrm{kDa}$, has a limited expression pattern virtually confined to the gastrointestinal tract. ${ }^{24,25}$ In the stomach, the CK20-positivity is situated in the upper part of the gastric pits. ${ }^{26}$ As CK20 is expressed throughout the whole gastro-intestinal tract, it is less useful than CK7, in order to distinguish metastasis of adenocarcinomas originating from different parts of the gastrointestinal tract. ${ }^{26}$ However the combined expression of both cytokeratins may solve this problem, as for example, metastases of colonic adenocarcinomas are negative for CK7 and positive for CK20, whereas the opposite is true for adenocarcinomas of the pancreas or biliary tract. ${ }^{27}$
In our study, we aimed to analyze the coexpression of CK7 and CK20 on three different tumor groups: esophageal, cardiac and distal gastric adenocarcinomas. In accordance with the study of Ormsby et $a l,{ }^{17}$ we observed that the CK7 + /CK20- pattern was significantly more prevalent in esophageal adenocarcinomas, than in distal gastric carcinomas, resulting in a comparable specificity (79 vs 69\%). Based on their study, Ormsby et a ${ }^{17}$ concluded that this cytokeratin pattern is confined to esophageal adenocarcinomas. In our study, we included cardiac adenocarcinomas. Immunohistochemical analysis showed that the CK7 + /CK20- cytokeratin pattern was not specific for esophageal adenocarcinomas, as the prevalence was similar in cardiac adenocarcinomas. This resulted in a low specificity of this CK7 + /CK20 - pattern in the distinction between these two types of adenocarcinomas of the gastroesophageal junction, namely $31 \%$. Based on this cytokeratin pattern, it was only possible to make a distinction between adenocarcinomas of the gastroesophageal junction vs distal gastric adenocarcinomas. However, this rarely poses a problem as the origin of the tumor can be determined based on the localization of the tumor.

Our data, however, are in contrast with the recently published study of Taniere et al, ${ }^{28}$ who showed that esophageal adenocarcinomas have a distinct cytokeratin pattern, different from cardiac adenocarcinomas. ${ }^{28}$ The CK7 + /CK20 - was highly specific for esophageal adenocarcinomas (76\%). In their study, cardiac adenocarcinomas were characterized by a variable cytokeratin pattern. This discrepancy in results can not be explained by a different immunohistochemical method as the same clones were used, applying an avidin-biotin technique with antigen retrieval. Moreover, the prevalence of the CK7 +, CK20- pattern in esophageal adenocarcinomas was not significantly different (74 vs $68 \%$ ) in spite of minor differences in inclusion criteria of distal esophageal adenocarcinomas (Taniere et al and our data) or Barrett adenocarcinomas (Ormsby et al). ${ }^{17,28}$ Another possible explanation is the differences in classification of the adenocarcinomas at the gastroesophageal junction as they subtyped their tumors according to the criteria of Siewert et $a .^{29}$ However, when we classified our tumors according to these criteria, the results of our study did not change (data not shown).

Distal gastric adenocarcinomas had a more variable staining pattern. In contrast to the gastroesophageal adenocarcinomas, the CK7-, CK20pattern is more characteristic for distal gastric adenocarcinomas. Although this pattern is present in only half of the population of distal gastric cancers, this cytokeratin pattern has a high specificity and moderately high sensitivity. These results are in contrast to the study of Ormsby et $a l^{17}$ in which no predominant staining pattern was found. In the study of Shen et $a l^{30}$ and Kende et al the majority of distal cancers showed a combined 
expression of cytokeratin 7 and 20 between 66 and $100 \%$. This prevalence is significantly higher than that in our study population (11\%), in which, however, our result is comparable with the data of Taniere et $a l^{28}(12 \%)$ and other publications. ${ }^{17,31}$

The histogenetical relationship between intestinal metaplasia and intestinal type of gastric adenocarcinomas and Barrett's adenocarcinomas is wellknown. Recently, Shen et $a l^{30}$ demonstrated a similar cytokeratin pattern in the gastric cancer and the intestinal metaplasia in the surrounding mucosa. With the exception of one case, their population only consisted of intestinal-type adenocarcinomas. However, we found a similar coexpression of these cytokeratins in intestinaland diffuse-type adenocarcinomas (Figure 4). Our results confirmed those of Goldstein et $a l^{32}$ and question the linking of cytokeratin expression to intestinal metaplasia and to cancer.

Our results are in fact a reflection of the published conflicting data on the cytokeratin pattern, found in association with Barrett's esophagus. According to Ormsby et $a l^{33,34}$ and Couvelard et al, ${ }^{35}$ Barrett's mucosa is characterized by a specific cytokeratin pattern, in which the CK7-positivity is situated in the superficial and deep glands of the intestinal metaplasia, whereas only the surface epithelium and superficial glands were positive for CK20. According to these authors, this staining pattern is highly specific for Barrett's esophagus and absent in gastric intestinal metaplasia. However, El-Zimaity and Graham ${ }^{36}$ and Glickman et $a l^{37}$ demonstrated both that this cytokeratin pattern is not restricted to the Barrett's esophagus, but is as frequently present in the intestinal metaplasia of the cardia. Moreover, both studies showed that, be it in a limited number $(14-27 \%)$, this cytokeratin pattern is present in gastric intestinal metaplasia. These data show similarities with the distribution pattern of cytokeratins in our study population.

In conclusion, although an accurate classification of the gastroesophageal adenocarcinomas is necessary in order to determine the incidence rate of these tumors and to evaluate the success of treatment in function of the chosen therapy, the determination of the origin of the tumor is hampered by the bulk of the tumor. Some studies defined the origin of esophageal adenocarcinomas by showing a unique coexpression of CK7 + and CK20-, which was absent in gastric tumors. ${ }^{17,30} \mathrm{Up}$ to a certain level our study confirm these data. These studies, however, were confined to tumors situated in the distal stomach. In contrast to these studies, we analyzed the expression pattern not only in distal gastric carcinomas but also in cardiac adenocarcinomas. The results of our study showed that the exact origin of tumors, situated at the gastroesophageal junction, as being an esophageal or cardiac tumor, cannot be determined by evaluation the coexpression of CK7 and CK20. Based on this cytokeratin expression pattern, it is not possible to make a distinction between an esophageal or cardiac adenocarcinomas.

\section{References}

1 Parkin DM, Pisani P, Ferlay J. Estimates of the worldwide incidence of eighteen major cancers in 1985. Int Cancer 1993;54:594-606.

2 Devesa SS, Blot WJ, Fraumeni JF Jr. Changing patterns in the incidence of esophageal and gastric carcinoma in the United States. Cancer 1998;83:2049-2053.

3 Blot WJ, Devesa SS, Kneller RW, et al. Rising incidence of adenocarcinoma of the esophagus and gastric cardia. JAMA 1991;265:1287-1289.

4 El-Serag HB, Mason AC, Petersen N, et al. Epidemiological differences between adenocarcinoma of the oesophagus and adenocarcinoma of the gastric cardia in the USA. Gut 2002;50:368-372.

5 Bruno L, Nesi G, Montinaro F, et al. Clinicopathologic findings and results of surgical treatment in cardiac adenocarcinoma. J Surg Oncol 2000;74:33-35.

6 De Manzoni G, Pedrazzani C, Pasini F, et al. Results of surgical treatment of adenocarcinoma of the gastric cardia. Ann Thorac Surg 2002;73:1035-1040.

7 Ellis FH, Heatley GJ, Krasna MJ, et al. Esophagogastrectomy for carcinoma of the esophagus and cardia: a comparison of findings and results after standard resection in three consecutive eight-year intervals with improved staging criteria. J Thorac Cardiovasc Surg 1997;113:836-848.

8 Maehara Y, Moriguchi S, Kakeji Y, et al. Prognostic factors in adenocarcinoma in the upper one-third of the stomach. Surg Gynecol Obst 1991;173:223-226.

9 Lerut T, Coosemans W, De Leyn P, et al. Reflections on three field lymphadenectomy in carcinoma of the esophagus and gastroesophageal junction. Hepatogastroenterology 1999;46:717-725.

10 Van de Ven C, De Leyn P, Coosemans W, et al. Threefield lymphadenectomy and pattern of lymph node spread in T3 adenocarcinoma of the distal esophagus and the gastro-esophageal junction. Eur J Cardiothorac Surg 1999;15:769-773.

11 Dixon MF, Neville PM, Mapstone NP, et al. Bile reflux gastritis and Barrett's oesophagus: further evidence of a role for duodenogastro-oesophageal reflux? Gut 2001;49:359-363.

12 Fass R, Hell RW, Garewal HS, et al. Correlation of oesophageal acid exposure with Barrett's oesophagus length. Gut 2001;48:310-313.

13 Csendes A, Smok G, Burdiles P, et al. Carditis an objective histological marker for pathologic gastroesophageal reflux disease. Dis Esoph 1998;11:101-105.

14 Öberg S, Peters JH, DeMeester TR, et al. Inflammation and specialized intestinal metaplasia of cardiac mucosa is a manifestation of gastroesophageal reflux disease. Ann Surg 1997;226:522-532.

15 Chen Y-Y, Antonioli DA, Spechler SJ, et al. Gastroesophageal reflux disease versus Helicobacter pylori infection as the cause of gastric carditis. Mod Pathol 1998;11:950-956.

16 Goldblum JR, Vicari JJ, Falk GW, et al. Inflammation and intestinal metaplasia of the gastric cardia: The role of gastroesophageal reflux and $H$. pylori infection. Gastroenterology 1998;114:633-639.

17 Ormsby AH, Goldblum JR, Rice TW, et al. The utility of cytokeratin subsets in distinguishing Barrett's-related 
oesophageal adenocarcinoma from gastric adenocarcinoma. Histopathology 2001;38:307-311.

18 Wittekind C, Henson DE, Hutter RVP, et al. TNM Supplement. A Commentary on Uniform Use, 2nd ed. Wiley-Liss: New York, 2001.

19 Sampliner RE. Practice guidelines in the diagnosis, surveillance, and therapy of Barrett's oesophagus. The Practice Parameters Committee of the American College of Gastroenterology. Am J Gastroenterol 1998;93:1028-1032.

20 Lauren P. The two histological main types of gastric carcinoma: diffuse and so-called intestinal-type carcinoma. An attempt at a histo-clinical classification. Acta Pathol Microbiol Scand 1965;64:31-49.

21 Weinstein WM, Ippoliti AF. The diagnosis of Barrett's esophagus: goblets, goblets, goblets. Gastrointes Endosc 1996;44:91-95.

22 Miettinen M. Keratin immunohistochemistry: update of applications and pitfalls. Pathol Annu 1993;28(Part 2):113-143.

23 Ramaekers F, Van Niekerk C, Poels L, et al. Use of monoclonal antibodies to keratin 7 in differential diagnosis of adenocarcinomas. Am J Pathol 1990;136: 641-655.

24 Moll R, Franke WW, Schiller DL, et al. The catalog of human cytokeratins: patterns of expression in normal epithelia, tumors and cultured cells. Cell 1982;31: 11-24.

25 Moll R, Löwe A, Laufer J, et al. Cytokeratin 20 in human carcinomas. A new histodiagnostic marker detected by monoclonal antibodies. Am J Pathol 1992;140:427-447.

26 Miettinen M. Keratin 20. Immunohistochemical marker for gastrointestinal, urothelial, and Merkel cell carcinomas. Mod Pathol 1995;8:384-388.

27 Tot T. Adenocarcinomas metastatic to the liver. The value of cytokeratins 20 and 7 in the search for unknown primary tumors. Cancer 1999;85:171-177.

28 Taniere P, Borghi-Scoazec G, Saurin JC, et al. Cytokeratin expression in adenocarcinomas of the esopha- gogastric junction: a comparative study of adenocarcinomas of the distal esophagus and of the proximal stomach. Am J Surg Pathol 2002;26:1213-1221.

29 Siewert JR, Stein HJ. Adenocarcinoma of the gastroesophageal junction: classification, pathology and extent of resection. Dis Esoph 1996;9:173-182.

30 Shen B, Ormsby AH, Dumot JA, et al. Cytokeratin expression patterns in noncardia intestinal metaplasiaassociated gastric adenocarcinoma. Implication for the evaluation of intestinal metaplasia and tumors at the esophagogastric junction. Cancer 2002;94:820-831.

31 Chu P, Wu E, Weiss LM. Cytokeratin 7 and cytokeratin 20 expression in epithelial neoplasms: a survey of 435 cases. Mod Pathol 2000;13:962-972.

32 Goldstein NS, Long A, Kuan SF, et al. Colon signet ring cell adenocarcinomas: immunohistochemical characterization and comparison with gastric and typical colon adenocarcinomas. Appl Immunohistochem Mol Morphol 2000;8:183-188.

33 Ormsby AH, Goldblum JR, Rice TW, et al. Cytokeratin subsets can reliably distinguish Barrett's esophagus from intestinal metaplasia of the stomach. Hum Pathol 1999;30:288-294.

34 Ormsby AH, Vaezi MF, Richter JE, et al. Cytokeratin immunoreactivity patterns in the diagnosis of shortsegment Barrett's oesophagus. Gastroenterology 2000;119:683-690.

35 Couvelard A, Cauvin J-M, Goldfain D, et al. Cytokeratin immunoreactivity of intestinal metaplasia at normal oesophagogastric junction indicates its aetiology. Gut 2001;49:761-766.

36 El-Zimaity HMT, Graham DY. Cytokeratin subsets for distinguishing Barrett's esophagus from intestinal metaplasia in the cardia using endoscopic biopsy specimens. Am J Gastroenterol 2001;96:1378-1382.

37 Glickman JN, Wang H, Das KM, et al. Phenotype of Barrett's esophagus and intestinal metaplasia of the distal esophagus and gastroesophageal junction. An immunohistochemical study of cytokeratins 7 and 20, Das-1 and 45MI. Am J Surg Pathol 2001;25:87-94. 